\title{
AUTO SPRUCE TRIAL SYSTEM (ASTS)
}

Huma Hassan Rizvi

Computer Engineering Department Sir Syed University of Engineering and Technology, Karachi, (Pakistan)

E-mail: humahrizvi@yahoo.com

Sana

Software Engineering Department Sir Syed University of Engineering and Technology, Karachi, (Pakistan)

E-mail: raosaleem770@gmail.com

Dr. Sadiq Ali khan

Department of Computer Science University of Karachi, (Pakistan) E-mail: msakhan@uok.edu.pk

Muhammad Khurrum Department of Informatics Malaysia university of science \& technology, (Malaysia) E-mail: muhammad.khurram@pg.must.edu.my

Khalique Ahmed Computer Engineering Department Sir Syed University of Engineering \& Technology, Karachi, (Pakistan) E-mail: kkhanzada@hotmail.com 


\section{ABSTRACT}

Auto Spruce Trial System (ASTS) is designed to provide a platform in which children's intelligence and cognitive behavior are tested through Wechsler Intelligence Scale for Children (WISC). This test is used for a children assessment and find out their abilities learning and disabilities, as well as a clinical device. ASTS system is an automated testing system which conducts the children test and generates their intelligence result automatically. We automate the system as mentioned above in Pakistan which is taken manually and consumes a lot of time. It does not really matter how much intelligence one has, what makes a difference is the manner by which well one uses his/her intelligence. This test is applicable for those children's whose parents are worried about their mental health's issues and their learning potential. An intelligence test can encourage guardians and instructors make judgments around an individual child's educational course, standard, or in need of special education.

\section{KEYWORDS}

Wechsler Intelligence Scale for Children (WISC).

\section{INTRODUCTION}

This testing system as mentioned above, developed by David Wechsler. It's a separately directed intelligence test for children between the ages of six and sixteen. The original test as mentioned above was developed in 1939, and this test divided into several of the subtests. The subtest was arranged into Verbal and Performance scales. These test scores based on:

- Verbal IQ(VIQ)

- Performance IQ(PIQ)

- Full Scale IQ (FSIQ)

The third edition was published in 1991 named as WISC-III. This edition has introduced a new subtest as a measure of processing speed. These four new index scores were introduced to represent more narrow domains of cognitive function:

- Verbal Comprehension Index (VCI)

- Perceptual Organization Index (POI)

- Freedom from Distractibility Index (FDI)

- Processing Speed Index (PSI)

The WISC-IV and WISC-V are published in the year 2003 and 2014 respectively. The WISC-V has included a total of 21 subtests which based on 15 composite scores [2].

In this paper we talk about a sort of use named as ASTS which can assist guardians with making a brilliant and prosperous future for their kids.

The application ASTS can be utilized in schools for special children's. ASTS is used not only as an intelligence test, but it is also used for other indicative purposes. IQ scores detailed by the ASTS and these outcomes can be utilized as a component to diagnose the children mental retardation and specific learning disabilities. But, here in this application we are just focusing on how to find out the cognitive functioning of a child. 


\subsection{Purpose}

ASTS is quite different from others. It is more reliable than any other system and Institutionalized knowledge tests are developed by strict rules to guarantee unwavering quality and legitimacy. This test is reliable when achieved a desired outcome. The main purpose of this system is to provide guidance to the parents who's really concerned about their children's mental health issues, so it will be a good approach to create something new and more reliable.

\subsection{Scope}

Since, we know that it is a fact that everybody wants a new idea or something innovative. Auto Spruce Trial System (ASTS) is designed for the diagnosis of the Intelligence Quotient (IQ) level of a child and will be able to predict the presence of disorder in the children based on the age and number of answered question in a specific time and patterns based on the scaling system. By using ASTS, we can easily determine the cognitive functioning of any child. This testing system as mentioned above is applicable for children from age six to sixteen years. This test is utilized as an intelligence test, as well as a clinical apparatus. This project utilizes all our work, academic skills and our experience to making a remarkable source for us to learn more things and grow more into this field.

\subsection{Modules of auto spruce trial system}

Our system consists of 3 modules, as follows:

Module-1: Pre-designed Testing System

Module-2: Consultancy

Module-3: Bulletin Board

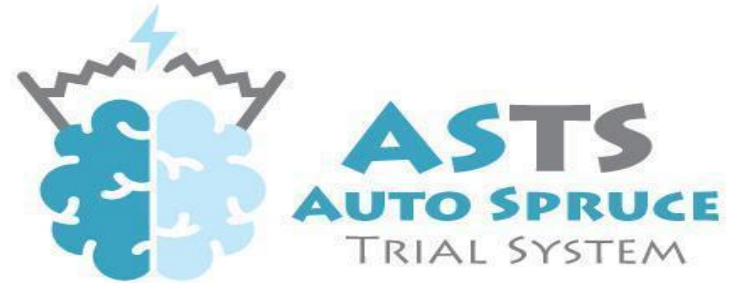

Figure 1. Logo of our application.

\section{Module-1: Pre-designed Testing System}

In this module, we simply automate the testing system as mentioned above it's allow the physiatrist to identify the stages of mind development. It has four main indexes. These indexes are below:

i) Verbal Comprehension Index (VCI)

ii) Perceptual Reasoning Index (VRI)

iii) Working Memory Index (WMI)

iv) Processing Speed Index (PSI)

There are a variety of subtests within each of these indexes[3].

1. The VCI Score test your child's intelligence and knowledge. The subtest includes:

- Vocabulary, Similarities, Comprehension, Information*, Word Reasoning*

2. The PRI scores is related to intelligence and ability to learn new information. The subtest includes:

- Block Design, Matrix Reasoning, Picture Concepts, Picture Completion* 
3. WMI score is related to short term memory. The subtest includes:

- Forward Digit Span, Backward Digit Span, Letter-Number Sequencing, Arithmetic*

4. PSI test focuses on mental quickness and task performance, its mainly concerned with concentration and attention. The subtest includes:

- Coding, Symbol Search, Cancellation* [3].

\section{Module-2: Consultancy}

It provides a consultancy section. Everyone who gives test, do not understand their test result and for their easiness we will provide consultants who will guide them online and evaluate their personality perfectly.

\section{Module-3: Bulletin Board}

Bulletin Board includes a portion where users can view updates about a particular issue or topic. It is a surface intended for the posting of public messages. It displays the daily updates of a website. If anything new happens, will be shown at the bulletin board.

\section{LITERATURE REVIEW}

Auto spruce trial system is an automated intelligence testing system which is based on the real and authorized data bank set. Automated System and websites exists, that conduct WISC-IV testing but their data set is not appropriate and according to the measures of testing system as mentioned above credibility. ASTS applies accurate and authenticate data set based on level of WISC-IV. ASTS is quite different from others. ASTS, is more reliable than any other system and standardized intelligence tests. ASTS is built by strict rules to ensure reliability and validity. A test result is considered reliable if we can get the equivalent/comparative outcome over and over.

ASTS is designed to overcome the difficulties of psychologists when they take manual tests and generate their result in few days. The difficulties, they face are:

1. The test is taken manually.

2. The result is generated lately.

3. Proper time is not given to individuals.

\subsection{Old methods used}

\section{A Test Sheet Algorithm for Assessments}

A dynamic programming approach is used to solve the problem generated by multiple criteria test-sheet. This utilizes the techniques of clustering and dynamic programming that allows the construction of a possible test sheet in accordance with the specified requirements. In this paper some experimental results and the testsheet-generating strategy of ITED is discussed to evaluate the efficiency of the approach [5].

\section{Fuzzy Logic-based Student Learning Assessment Model}

In this article a diagnosis model based on fuzzy logic has been presented. One of the main advantages of this model is that it sanctions for a representation of interpret able cognizance since it is predicated on rules when the reasoning is well defined as well as when the reasoning is intuitive, as a result of experience. The qualitative and quantitative criteria in student assessment proposed by the teachers 
can be easily improved (linguistic variables as well as fuzzy rules) adding a high degree of flexibility [11].

In Development of Computer-Assisted Testing System with Genetic Test Sheet technique is followed [12]. They proposed two genetic algorithms:

- CLFG

- FIFG

Above techniques are used for test sheet-generating problems. In a less time by applying these approaches we can get the test sheets with near-optimal discrimination degrees. The two algorithms have been embedded in a CAI system, Intelligent Tutoring, Evaluation, and Diagnosis that provides the easiness and the more informative tool for the instructors an learners. The (ITED-II) testing sub systems generate the test sheets by accepting the assessment requirements by reading the test items from the item banks. In the end the test results are sending to the tutoring sub system for the arrangements of adaptive subject materials [12].

\section{Generation Algorithm for Test Sheet Results}

The test sheet generating issues are solved through an adaptive cellular genetic algorithm, which is based on selection strategy. This algorithm is a combination of Adaptive Test Sheet Generation and cellular genetic algorithm. This approach resolves the problems of test sheet generation space, improves the fitness of test sheet and also improves the assessment of child's. These techniques also improve the accuracy in calculations and convergence speed of calculations in test sheet generation [13].

\section{An Evolutionary Intelligent Water Drops Approach for Intelligence Test sheet Results Generation}

In this paper, an intelligence test sheet result generating problems and issues are resolved. The computerized test sheet results with multiples assessments and calculations are one of the major issues in the Computer Assisted Testing System an E-Learning technology. A huge and verity of different tests, questions and task banks with different abilities are involved in the assessments test, even randomized test cannot serve the purpose of assessment and cannot generate an accurate output. The accurate result of the system is based on correct question bank and algorithm. It is difficult to develop the assessment sheet that satisfies the all assessment criteria. Evolutionary Intelligent Water Drops is best and more suitable algorithm which solves all the issues related to test sheet results and also solve the huge amount of question bank assessments test [14].

\section{Genetic Algorithm used for assessment test}

In this research, genetic algorithm approach is used for genetic assessment test. This method is used for optimized the sequences multiple variety and group of tests which have used for same purpose and it is also use a less amount of hardware resources for optimal solutions. These tests are time consuming and some restrictions are applied. In this approach representative keywords used for a particular test. This approach has three major elements:

- Teaching

- Learning 
- Evaluation

The genetic algorithm helps in finding the best appropriate solutions [15].

The reference is given below of a report that is a result of testing tool kit as mentioned above of a child it describes all the tables that are used for performance evaluation [1][4].

\section{METHODOLOGY}

\subsection{Method/Technique}

The main motive of our development is to produce precise and trustworthy results. We don't have the right to ruin anyone's life as it is a matter of very serious problem, therefore the results of the system must be reliable. There have been many approaches that drive different results based on decisions that are made on different states. In every stage, a decision which achieves a reward closer to the total rewards is desirable. The new approach adopts fuzzy logic theory to diagnose the difficulty level of test items, in accordance with the learning status and personal features of each student, and then applies the techniques to the test sheet construction. Clustering and dynamic programming is also an approach to solve such issues. ASTS system is an automated testing system which conducts the children test and generate their intelligence result automatically in which we will apply fuzzy logic instead of clustering techniques and dynamic programming approach. We automate the testing system as mentioned above in Pakistan which is taken manually and consumes a lot of time. [4] ASTS will serve as an intelligent assistance to psychologist. The fuzzy logic will make the system more efficient and time saving and it will also become very helpful for the psychologist.

\subsection{Product Pers pective}

The system Auto Spruce Trial System (ASTS) is designed for the diagnosis of the Intelligence Quotient (IQ) level of a child and will be able to predict the presence of disorder in the children based on the age and number of answered question in a specific time and patterns based on the scaling system or the implementation of algorithm that are to be decided. The scores are cross matched with the scaled scores, composite scores, percentile rank or algorithm and provide the result in the terms of perfection or disorder. Type, kind, level and seriousness of disorder will be further provided in the consultancy section if required.

\subsection{System Functions}

The functions of the system are as follows:

- Generation of questions from question bank.

- Make record for the answered question.

- Compare the answers by the engine and perform calculations.

- Predict the IQ level.

- Predict disorder in the child if present on behalf of his/her answers.

\subsection{User View}

- User must know about this application features and a basic knowledge to operating the internet. 
- User of this is generally the children that will interact so only their proper attention is required.

- This system can also be used by parents/system for the result tracking of the child so; they should have basic knowledge of computer.

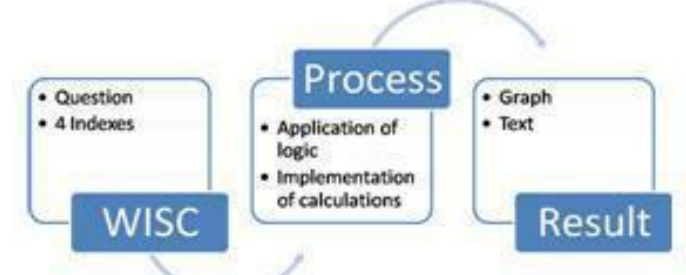

Figure 2. Abstract view of system.

\subsection{Operating Environment}

This is a web and android based system and hence will require a good GUI for good results. The basic need is the browser version for web users and android version for android user.

\subsection{Constraint}

- Expertise of members in the software used can be a constraint for the timely completion of the system.

- Inappropriate working of database and interface may be a constraint.

- Internet connection is important to run the function of the application.

- Database is shared between both web and mobile application it may be forced to queue incoming requests and therefore increase the time it takes to fetch data.

\subsection{System Assumption}

- Great amount of memory is required in cell phones to use this system.

- If your cell phone is not supporting well in memory and proper hardware resources so you can't access this system.

\subsection{User Role}

- First step is the users register him/her self into the system.

- The second step is system provides the access key to users.

- The third step is according to the age level of user the system starts to show the test questions in order to take test of user then user start to give the test.

- Next step is when user completed the test the system will show the test results. The result is in percentage form which determines the level of user's intelligence and their cognitive abilities.

- The last step is the user logout from the system.

\subsection{Overall System working through Diagram}

Overall working of the system through diagram is as follows: 


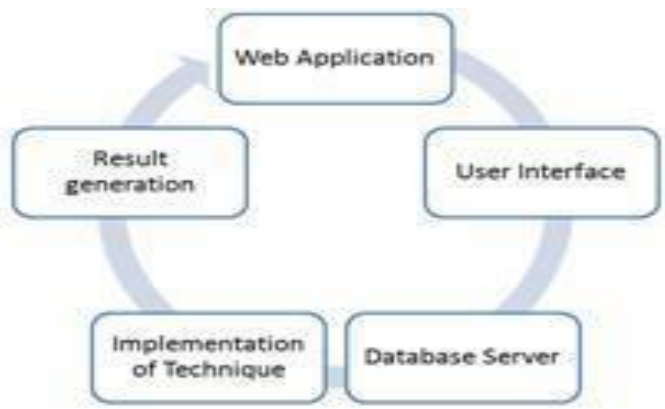

Figure 3. Web view of system.

Figure 4. android view of system.

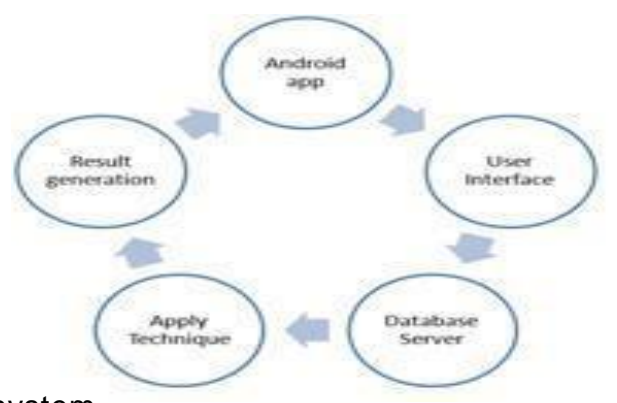

\subsection{Hardware and Software}

\section{Hardware}

- Laptop Mobile

- Operating Systems

\section{Windows Android}

- Databases

- MySql SQLite

Programming Languages

- Java PHP

- HTML5 CSS3

- Bootstrap

- JSON

\section{RESULTS AND DISCUSSIONS}

We automate the testing system as mentioned above in Pakistan which is taken manually and consumes a lot of time. Psychologists complete this test in 2/3 days or in an entire week period of time. They cannot take this test continuously because neither they can concentrate on a test after 2 nor 3 hours nor children will be able to give test continuously.

The manually system is totally converted into automated system. It stands and outmost the credibility level of all available websites conducting WISC-IV tests. Its output/result will be a score sheet and recommendations paper of child's intelligence for parents and Psychologists. 


\section{Comparison:}

\begin{tabular}{|c|c|c|c|c|}
\hline Websites & Reliable & Correct & Usability & Authentic \\
\hline Assessment.com[6] & No & Yes & No & No \\
\hline Quiznatic.com[7] & No & Yes & No & No \\
\hline Fundaction.com[8] & No & No & No & No \\
\hline Brainmetrix.com[9] & No & No & No & No \\
\hline Asts4childern.com[10] & Yes & Yes & Yes & Yes \\
\hline
\end{tabular}

Table 1. Comparison of ASTS system with other systems $[6,7,8,9,10]$.

The comparison can be easily understandable through this chart:
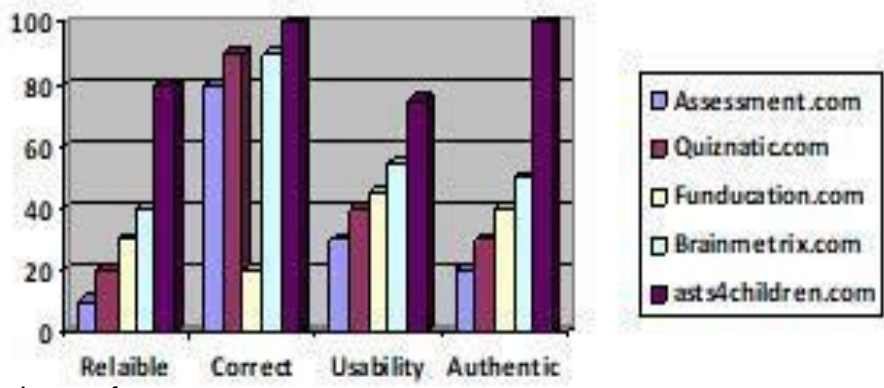

Figure 5. Comparison of system.

\section{CONCLUSION}

In this research, we have presented an application by which psychologists can take their assessment test easily. They don't have to wait for the entire week for result generation. Even, they don't have to do calculations on their own. All they have to do is to check the behavior of the children and help them through queries if they are stuck. The rest of the work will be done by the system itself, which includes calculations, displaying questions and results generation. This will help kids in their primary ages, when they are studying. Not only kids but parents who are concerned about their children will get benefit too. The system will let parents know about their child weaknesses and IQ. Not only this, parents can also consult with the consultants about how to increase their children IQ and what should be done and what shouldn't be done. This will help children to make their future bright and prosperous. In future, this application can be made more user friendly by implementing different GUI. For now, this application is only FYPs demonstration. But, after interacting with superior psychologist if they allow this application to be used for clinical purpose then we will implement it in clinics. And, we will not stop here, sooner it will be implemented in Schools and other educational institutions.

\section{REFERENCES}

\subsection{Patent}

[1] A WISC Descriptive and Graphical Report by Michelle C. Rexach, Licensed School Psychologist, Florida Department of Health.

\subsection{Websites}

[2] http://www.school-psychology.com.au/blog/wechslerintelligence-scale-forchildren-wisc-iv/

[3] https://www.helloq.com/overview/the-q-interactive-library/wisc-iv.html 


\subsection{Conference Proceedings}

[4] Anne-Marie Kimbell, "An Overview of the WISC “, Ph.D. National Training Consultant Pearson, 2015.

[5] Gwo-Jen Hwang, "A Test-Sheet-Generating Algorithm for Multiple Assessment Requirements”, ieee transactions on education, vol. 46, no. 3, august 2003.

\subsection{Websites}

[6] http://assessment.com/

[7] http://www.quiznatic.com/

[8] http://funducation.com/

[9] http://brainmetrix.com/

[10] http://asts4children.com/

\subsection{Research paper}

[11] Constanza Huapaya1, “ Proposal of Fuzzy Logic-based Students Learning Assessment Model".

[12] Gwo-Jen Hwang, Bertrand M. T. Lin, Hsien-Hao Tseng, and Tsung-Liang Lin, "On the Development of a Computer-Assisted Testing System With Genetic Test Sheet-Generating Approach", ieee transactions on systems, man, and cyberneticspart c: applications and reviews, vol. 35, no. 4,november 2005.

\subsection{J ournal Article}

[13] Ankun Huang, Dongmei Li1, Jiajia Hou ,Tao Bi, “An Adaptive Cellular Genetic Algorithm Based on Selection Strategy for Test Sheet Generation", International Journal of Hybrid Information Technology, Vol.8, No.9 (2015).

[14] Kavitha, "Composition of Optimized Assessment Sheet with Multi-criteria using Evolutionary IntelligentWater Drops (EvIWD) Algorithm”, International Journal of Software Engineering and Its Applications, Vol. 10, No. 6 (2016).

[15] Doru Popescu Anastasiu, Nicolae Bold, and Daniel Nijloveanu, "A Method Based on Genetic Algorithms for Generating Assessment Tests Used for Learning",vol. 54, 2016, pp. 53-60. 